\title{
Preparation of pyridine-3,4-diols, their crystal packing and their use as precursors for palladium-catalyzed cross-coupling reactions
}

\author{
Tilman Lechel, Irene Brüdgam and Hans-Ulrich Reissig*
}

\section{Full Research Paper}

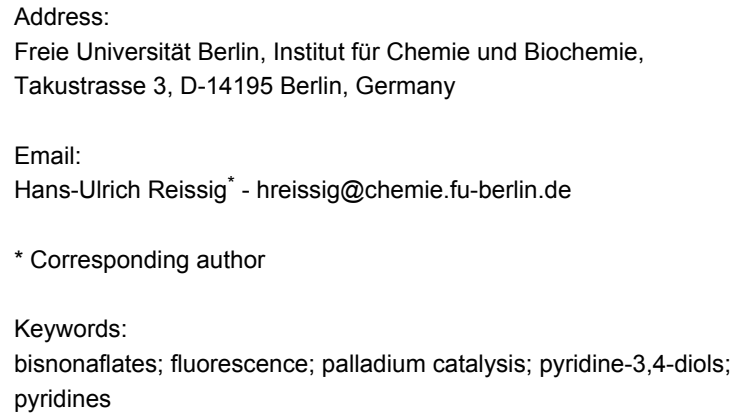

\author{
Beilstein J. Org. Chem. 2010, 6, No. 42. \\ doi:10.3762/bjoc.6.42 \\ Received: 23 February 2010 \\ Accepted: 09 April 2010 \\ Published: 29 April 2010 \\ Editor-in-Chief: J. Clayden \\ (C) 2010 Lechel et al; licensee Beilstein-Institut. \\ License and terms: see end of document.
}

\begin{abstract}
A series of trifluoromethyl-substituted 3-alkoxypyridinol derivatives has been deprotected to furnish pyridine-3,4-diol derivatives in good yields. The X-ray crystal structure analysis proved that a 1:1 mixture of pyridine-3,4-diols and their pyridin-4-one tautomers exist in the solid state. Subsequent conversion into bis(perfluoroalkanesulfonate)s were smoothly achieved. The obtained compounds were used as substrates for palladium-catalyzed coupling reactions. Fluorescence measurements of the biscoupled products showed a maximum of emission in the violet region of the spectrum.
\end{abstract}

\section{Introduction}

Pyridine scaffolds have been found in numerous naturally occurring compounds and are also frequently used in functional materials [1-4]. Pyridindiol derivatives are of particular interest as building blocks for the construction of dendritic nanostructures in supramolecular chemistry [5], whereas N-protected pyridine-3,4-diols find applications as potent chelating agents in medicinal chemistry [6]. Furthermore, perfluorinated heteroaromatic compounds are interesting synthetic intermediates for the development of novel pharmaceuticals [7]. Continuing our research on heterocyclic chemistry based on alkoxyallenes [8-17], we focused on the synthesis of trifluoro- methyl-substituted pyridine derivatives [18-23]. Herein, we report different methods for the deprotection of a range of 3-alkoxypyridinols 1 to give pyridine-3,4-diols 2 and the corresponding tautomers $\mathbf{2}^{\prime}$. This equilibrium between pyridindiols and hydroxypyridinones will be thoroughly investigated in the solid state as well as in solution. Furthermore, subsequent transformations into bistriflate or bisnonaflate derivatives will be described, followed by palladium-catalyzed coupling reactions. The resulting biscoupling products are analysed with regard to their photophysical properties. 
<smiles>[R2]Oc1c([R2])nc(C(F)(F)F)c([R])c1O</smiles>

1<smiles>[R]c1nc(C(F)(F)F)c([R])c(O)c1O</smiles>

2<smiles>[R]c1[nH]c(C(F)(F)F)c([R])c(=O)c1O</smiles>

2'
1a: $\left(R^{1}=M e, R^{2}=B n^{a}, R^{3}=H\right)$
2a, $74 \%$
1b: $\left(R^{1}=t-B u, R^{2}=M e^{b}, R^{3}=P h\right)$
2b, $63 \%$
1c: $\left(\mathrm{R}^{1}=t-\mathrm{Bu}, \mathrm{R}^{2}=\mathrm{TMSE}^{\mathrm{c}}, \mathrm{R}^{3}=\mathrm{H}\right)$
2c, quant.
1d: $\left(R^{1}=P h, R^{2}=M e^{b}, R^{3}=P h\right)$
2d, $94 \%$

Scheme 1: Deprotection of 3-alkoxypyridinols 1 to pyridine-3,4-diols 2. aMethod a: $\mathrm{Pd} / \mathrm{C}, \mathrm{H}_{2}, \mathrm{MeOH}, \mathrm{rt}, 1 \mathrm{~d}$; b ${ }^{\mathrm{M}} \mathrm{Method} \mathrm{b:} \mathrm{BBr}_{3}, \mathrm{CH}_{2} \mathrm{Cl}_{2}, 0^{\circ} \mathrm{C}$ to rt, $1 \mathrm{~d}$; cMethod c: TFA: $\mathrm{CH}_{2} \mathrm{Cl}_{2}(1: 2), \mathrm{rt}, 1 \mathrm{~h}$.

\section{Results and Discussion}

The preparation of pyridine-3,4-diol derivatives as depicted in Scheme 1 succeeded by using highly substituted trifluoromethyl-substituted 4-hydroxypyridine precursors 1 that have been prepared in two steps from lithiated alkoxyallenes, nitriles and carboxylic acids [21]. It is noteworthy, that the respective protecting group at $\mathrm{C}-3$ of the pyridine core was originally incorporated with the alkoxyallene moiety. The mild cleavage of the benzyl-protected pyridine 1a to diol 2a was achieved by hydrogenolysis in the presence of catalytic amounts of palladium on charcoal. Methyl ethers such as $\mathbf{1 b}$ or $\mathbf{1 d}$ were cleaved by Lewis-acids. The (2-trimethylsilyl)ethyl-protected pyridine 1c was easily deprotected to diol $\mathbf{2 c}$ by a Brønsted acid such as TFA. In most cases, the corresponding pyridindiols $\mathbf{2 a}-\mathbf{d}$ were obtained in good yields (63\%-quant.).
NMR-measurements in $\mathrm{CDCl}_{3}$ showed that the obtained pyridine-3,4-diols 2 are in equilibrium with their pyridin-4-one tautomers $\mathbf{2}^{\prime}$. For instance, in case of $\mathbf{2 c}$ the equilibrium is strongly shifted to the pyridin-4-one $\mathbf{2} \mathbf{c}^{\prime}$ (ratio $\mathbf{2} \mathbf{c}: \mathbf{2} \mathbf{c}^{\prime}=30: 70$ ). This ratio could be completely shifted to the pyridine-3,4-diol side by a polar protic solvent such as methanol. Surprisingly, the X-ray crystal structure measurement of compound 2c [24] revealed that in the solid state a $1: 1$ ratio of diol and its pyridinone tautomer $\mathbf{2} \mathbf{c}^{\prime}$ is preferred. Figure 1 shows that two pyridine-3,4-diol molecules are in one plane with two pyridinone molecules in a perpendicular plane. The two alternating planes are connected by hydrogen bridges.

As a consequence of our ongoing interest in perfluoroalkyl sulfonate chemistry [25], we converted the two hydroxyl groups

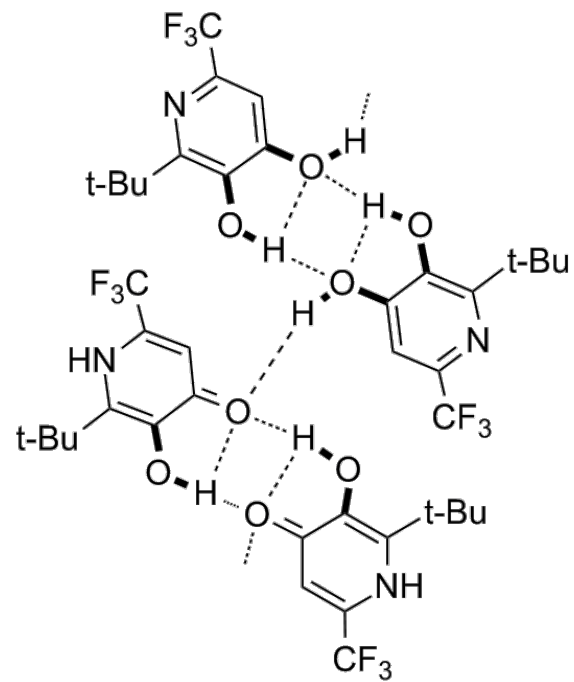

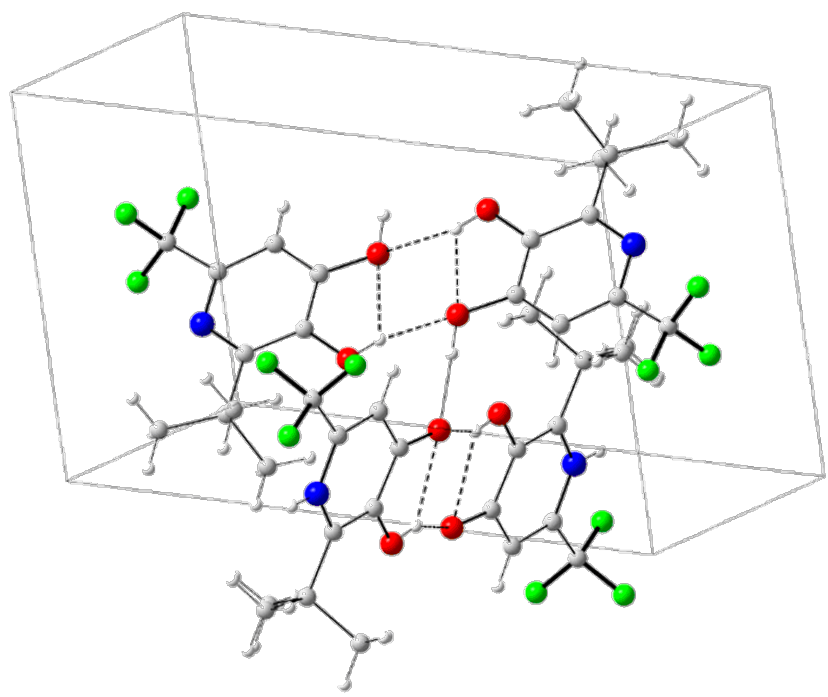

Figure 1: X-ray crystal structure of compound $2 \mathrm{c} / \mathbf{2} \mathrm{c}^{\prime}$. 
<smiles>[R]c1nc(C(F)(F)F)cc(O)c1O</smiles><smiles>C[13CH][13CH]</smiles>

2a: $\mathrm{R}^{1}=\mathrm{Me}$

2c: $R^{1}=t-B u$

2c: $R^{1}=t-B u$

2d: $R^{1}=P h$<smiles>[R20]Oc1c(OS(=O)O[R])cc(C(F)(F)F)nc1[R]</smiles>

3

3a: $\mathrm{R}_{\mathrm{f}}=\mathrm{CF}_{3}, 78 \%$

3b: $R_{f}=C F_{3}, 90 \%$

3c: $R_{f}=C_{4} F_{9}, 59 \%$

3d: $R_{f}=C_{3}, 47 \%$

Scheme 2: Conversion of pyridine-3,4-diols 2 into pyridinediyl bistriflates or -nonaflates 3. a) $\mathrm{Et}_{3} \mathrm{~N}, \mathrm{Rf}_{2} \mathrm{O}, \mathrm{CH}_{2} \mathrm{Cl}_{2}, 0{ }^{\circ} \mathrm{C}$ to $\mathrm{rt}, 1 \mathrm{~d}$.

into triflates or nonaflates, respectively (Scheme 2). These substituents represent very good leaving groups for subsequent functionalisations such as palladium-catalyzed $\mathrm{C}-\mathrm{C}$ cross-coupling reactions [26,27]. At first, the pyridindiols $\mathbf{2 a}$ and $\mathbf{2 c - d}$ were treated with $\mathrm{Et}_{3} \mathrm{~N}$ in dichloromethane and an excess of $\mathrm{Tf}_{2} \mathrm{O}$ or $\mathrm{Nf}_{2} \mathrm{O}$, respectively. This provided bistriflates or bisnonaflates $\mathbf{3 a}-\mathbf{d}$ as the only products in moderate to very good yields (47-90\%). A direct comparison showed that the treatment of $2 \mathbf{c}$ with $\mathrm{Tf}_{2} \mathrm{O}$ led to a higher yield than that with $\mathrm{Nf}_{2} \mathrm{O}$. This may be due to lower steric hindrance in the case of the triflating reagent.

As typical examples of possible palladium-catalyzed crosscouplings we performed several Sonogashira-reactions [28-30]. As described in Scheme 3, the pyridinyl-bistriflates or -nonaflates 3 were coupled with alkynes like phenylacetylene or (triisopropylsilyl)acetylene using $\mathrm{Pd}\left(\mathrm{PPh}_{3}\right)_{4}$ or alternatively,

$\mathrm{Pd}(\mathrm{OAc})_{2} / \mathrm{PPh}_{3}$ as catalyst and $\mathrm{CuI}$ as co-catalyst in the presence of a 1:2 mixture of $i \mathrm{Pr}_{2} \mathrm{NH}$ and DMF. Only the corresponding biscoupled products 4 , which were isolated in moderate yields (30-44\%) have been observed. Comparing entries 2 and 3, the coupling of an alkyne with a bisnonaflate gave a slightly lower yield than that with the corresponding bistriflate. A subsequent cleavage of the triisopropylsilyl group with a fluoride source provided product $\mathbf{5}$ in $58 \%$ yield.

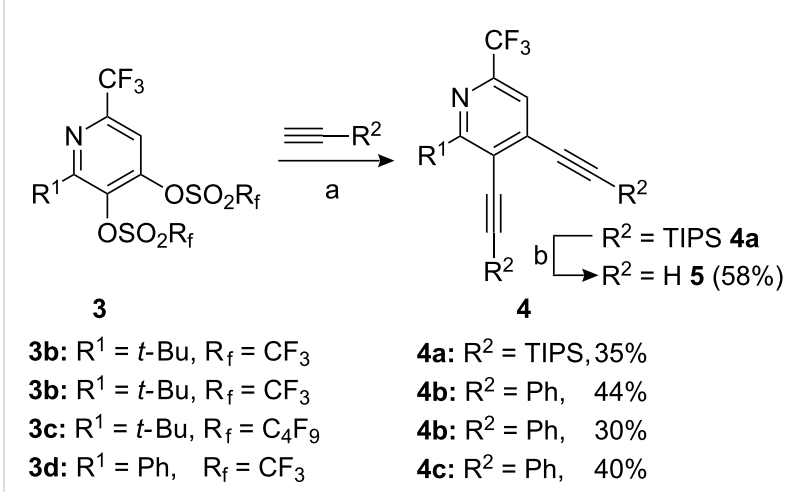

Scheme 3: Sonogashira couplings of pyridinediyl bis(perfluoroalkanesulfonates) 3. a) $\mathrm{Pd}\left(\mathrm{PPh}_{3}\right)_{4}$ [or $\mathrm{Pd}(\mathrm{OAc})_{2} / \mathrm{PPh}_{3}$, Cul, $\mathrm{Pr}_{2} \mathrm{NH}, \mathrm{DMF}, 70^{\circ} \mathrm{C}, 4$ h. b) TBAF, THF, rt, $1 \mathrm{~h}$.

The biscoupling reaction led to extended $\pi$-systems which might have interesting photophysical properties [31-33]. Hence, absorption and emission of $\mathbf{4 b}$ and $\mathbf{4 c}$ were studied. The results are depicted in Figure 2 and show absorption maxima in the range of 275-295 $\mathrm{nm}$ whereas the emission maxima are located between $385-400 \mathrm{~nm}$.

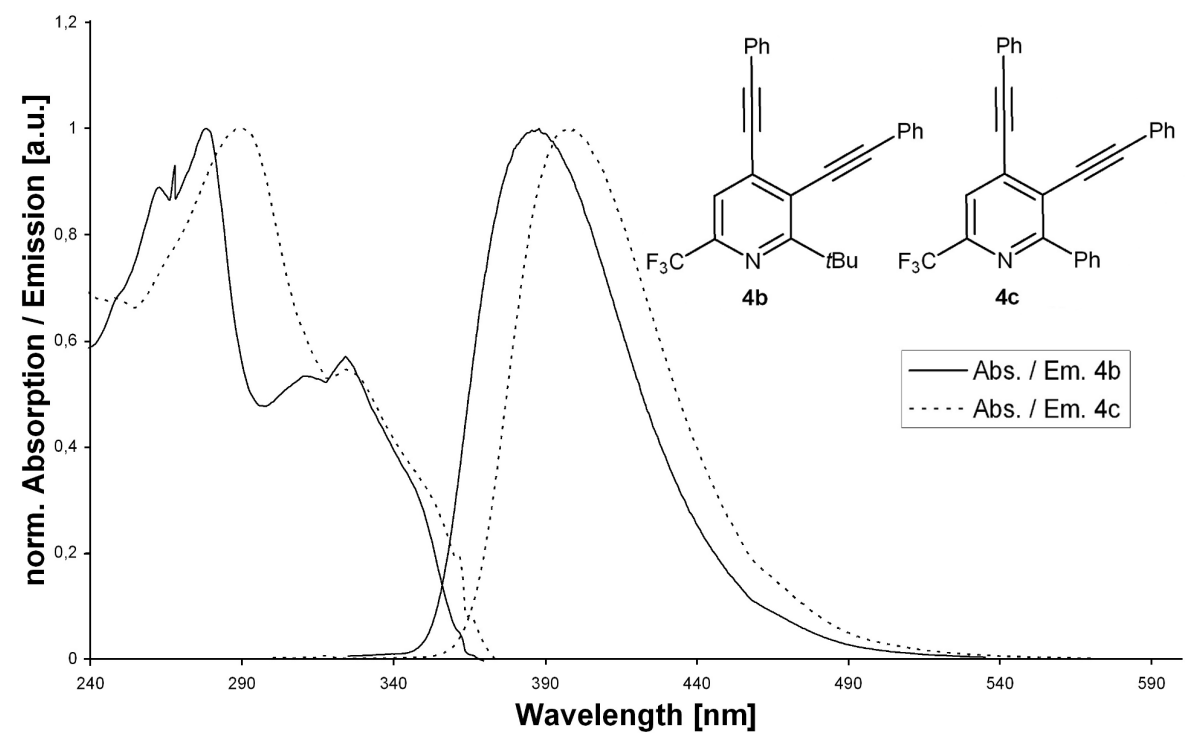

Figure 2: Absorption and fluorescence spectra of compounds $\mathbf{4 b}$ and $\mathbf{4 c}$. 
Both products are fluorescent, emitting light in the violet region and show similar Stokes shifts. Owing to the additional phenyl substituent at C-2 of pyridine $4 \mathbf{c}$, the $\pi$-system is slightly more extended and obviously influences the absorption and emission maxima with a bathochromic shift of $10 \mathrm{~nm}$.

\section{Conclusion}

In conclusion, we have successfully demonstrated that 3-alkoxypyridines are ideal precursors for the synthesis of pyridine-3,4-diol derivatives. The coexistence of pyridindiol and pyridinone tautomers in the solid state was discovered by an X-ray structure analysis. It was shown that pyridine-3,4-diols could easily be converted into bis(perfluoroalkanesulfonates) which represent substrates for the construction of extended $\pi$-systems using palladium-catalyzed coupling reactions. Moreover, compounds $\mathbf{4 b}-\mathbf{c}$ show interesting photophysical properties that might be thoroughly investigated in the future. The 3,4-dialkynyl-pyridine derivatives $\mathbf{4}$ or 5 are also candidates for Bergman cyclizations [34,35] which may establish a route to isoquinoline derivatives.

\section{Experimental}

\section{Deprotection of 3-alkoxypyridin-4-ols 1 , typical procedures}

Cleavage of the benzyloxy group by hydrogenolysis

A mixture of 1a $(970 \mathrm{mg}, 3.43 \mathrm{mmol})$ and palladium (365 mg, 10\% on charcoal, $0.34 \mathrm{mmol}$ ) in methanol $(6 \mathrm{~mL}$ ) was stirred for one day under an atmosphere of hydrogen. Filtration of the reaction mixture through celite with methanol afforded $489 \mathrm{mg}$ (74\%) of $\mathbf{2 a}$ as a colorless solid, $\operatorname{mp} 216{ }^{\circ} \mathrm{C}$.

2-Methyl-6-(trifluoromethyl)pyridine-3,4-diol (2a): ${ }^{1} \mathrm{H}$ NMR $\left(\mathrm{CD}_{3} \mathrm{OD}, 500 \mathrm{MHz}\right): \delta=2.41(\mathrm{~s}, 3 \mathrm{H}, \mathrm{Me}), 7.00(\mathrm{~s}, 1 \mathrm{H}, 5-\mathrm{H})$ ppm. OH-signals could not be detected. ${ }^{13} \mathrm{C}$ NMR $\left(\mathrm{CD}_{3} \mathrm{OD}\right.$, $126 \mathrm{MHz}): \delta=17.7$ (q, Me), $108.0(\mathrm{~d}, \mathrm{C}-5), 123.1$ (q, ${ }^{1} J_{\mathrm{CF}}=$ $273 \mathrm{~Hz}, \mathrm{CF}_{3}$ ), 139.1 (q, $\left.{ }^{2} J_{\mathrm{CF}}=35.3 \mathrm{~Hz}, \mathrm{C}-6\right), 139.0,144.4$, 154.1 (3 s, C-2, C-3, C-4) ppm. IR (KBr): $v=3350-3240(\mathrm{O}-\mathrm{H}$, $\mathrm{N}-\mathrm{H}), 3110-3040(=\mathrm{C}-\mathrm{H}), 3000-2670(\mathrm{C}-\mathrm{H}), 1650-1550(\mathrm{C}=\mathrm{O}$, $\mathrm{C}=\mathrm{C}) \mathrm{cm}^{-1}$. HRMS (ESI-TOF) calcd. for $\mathrm{C}_{12} \mathrm{H}_{9} \mathrm{~F}_{3} \mathrm{NO}_{2}$ $[\mathrm{M}+\mathrm{H}]^{+}:$194.0423, found: 194.0419. $\mathrm{C}_{7} \mathrm{H}_{6} \mathrm{~F}_{3} \mathrm{NO}_{2}$ (193.1): calcd. C, 43.53; H, 3.13; N, 7.25; found: C, 43.94; H, 3.10; N, 6.95 .

\section{Cleavage of the methoxy group by $\mathrm{BBr}_{3}$}

To a solution of $\mathbf{1 b}(370 \mathrm{mg}, 1.14 \mathrm{mmol})$ in dichloromethane (4 mL) under an argon atmosphere, $\mathrm{BBr}_{3}\left(1 \mathrm{M}\right.$ in $\mathrm{CH}_{2} \mathrm{Cl}_{2}$, $3.42 \mathrm{~mL}, 3.42 \mathrm{mmol}$ ) was added dropwise at $0{ }^{\circ} \mathrm{C}$ and allowed to warm to room temperature. The reaction was monitored by TLC; upon completion, ice-water was added and the mixture was extracted three times with dichloromethane $(5 \mathrm{~mL})$. The combined organic phases were dried over $\mathrm{Na}_{2} \mathrm{SO}_{4}$ and concentrated to dryness. Column chromatography on silica gel (hexane/ethyl acetate $=4: 1)$ afforded $222 \mathrm{mg}(63 \%)$ of $\mathbf{2 b}$ as a colorless solid, mp $192{ }^{\circ} \mathrm{C}$.

2-tert-Butyl-5-phenyl-6-(trifluoromethyl)pyridine-3,4-diol (2b): ${ }^{1} \mathrm{H}$ NMR $\left(\mathrm{CD}_{3} \mathrm{OD}, 500 \mathrm{MHz}\right): \delta=1.45(\mathrm{~s}, 9 \mathrm{H}, t-\mathrm{Bu}), 7.24-7.47$ $(\mathrm{m}, 5 \mathrm{H}, \mathrm{Ph}) \mathrm{ppm}$. OH-signals could not be detected. ${ }^{13} \mathrm{C}$ NMR $\left(\mathrm{CD}_{3} \mathrm{OD}, 101 \mathrm{MHz}\right): \delta=28.8,38.6(\mathrm{q}, \mathrm{s}, t-\mathrm{Bu}), 124.7$ (s, C-5), $126.3\left(\mathrm{q},{ }^{1} J_{\mathrm{CF}}=274 \mathrm{~Hz}, \mathrm{CF}_{3}\right), 129.3,129.4,131.5,133.6(3 \mathrm{~d}$, $\mathrm{s}, \mathrm{Ph}), 138.6\left(\mathrm{q},{ }^{2} J_{\mathrm{CF}}=32.3 \mathrm{~Hz}, \mathrm{C}-6\right), 144.3,151.1,154.3(3 \mathrm{~s}$, $\mathrm{C}-2, \mathrm{C}-3, \mathrm{C}-4) \mathrm{ppm}$. IR (KBr): 3450-3260 (O-H, N-H), 3085-3060 (=C-H), 3040-2880 (C-H), 1655-1585 (C=O, C=C) $\mathrm{cm}^{-1}$. HRMS $\left(80 \mathrm{eV}, 90{ }^{\circ} \mathrm{C}\right) \mathrm{m} / z$ calcd. for $\mathrm{C}_{16} \mathrm{H}_{16} \mathrm{~F}_{3} \mathrm{NO}_{2}$ : 311.11331; found: 311.11266 .

\section{Cleavage of the (2-trimethylsilyl)ethoxy group with TFA}

Pyridine derivative 1c (90 mg, $0.268 \mathrm{mmol}$ ) was dissolved in a 1:5 mixture of trifluoroacetic acid and dichloromethane $(3 \mathrm{~mL})$ and stirred for $1 \mathrm{~h}$ at room temperature. After the addition of water and dichloromethane $(5 \mathrm{~mL})$ the layers were separated and the aqueous layer was extracted twice with dichloromethane $(8 \mathrm{~mL})$. The combined organic layers were dried over $\mathrm{Na}_{2} \mathrm{SO}_{4}$ and concentrated to dryness. Column chromatography ( of $\mathbf{2 c}$ and $\mathbf{2} \mathbf{c}^{\prime}$ in a ratio of $30: 70$ as a colorless solid, $\mathrm{mp} 102-103{ }^{\circ} \mathrm{C}$.

2-tert-Butyl-6-(trifluoromethyl)pyridine-3,4-diol (2c): ${ }^{1} \mathrm{H}$ NMR $\left(\mathrm{CDCl}_{3}, 500 \mathrm{MHz}\right): \delta=1.43(\mathrm{~s}, 9 \mathrm{H}, t-\mathrm{Bu}), 7.11(\mathrm{~s}, 1 \mathrm{H}, 5-\mathrm{H})$ ppm. OH-signals could not be detected. ${ }^{13} \mathrm{C} \mathrm{NMR}\left(\mathrm{CDCl}_{3}, 126\right.$ $\mathrm{MHz}): \delta=28.2,37.6$ (q, s, $t-\mathrm{Bu}), 106.5$ (d, C-5), 115.6 (q, ${ }^{1} J_{\mathrm{CF}}$ $\left.=294 \mathrm{~Hz}, \mathrm{CF}_{3}\right), 132.2\left(\mathrm{q},{ }^{2} J_{\mathrm{CF}}=37.0 \mathrm{~Hz}, \mathrm{C}-6\right), 142.2,150.7$, 154.6 (3 s, C-2, C-3, C-4) ppm.

2-tert-Butyl-3-hydroxy-6-(trifluoromethyl)pyridin-4(1H)-one $\left(\mathbf{2 c}^{\prime}\right):{ }^{1} \mathrm{H} \mathrm{NMR}\left(\mathrm{CDCl}_{3}, 500 \mathrm{MHz}\right): \delta=1.51(\mathrm{~s}, 9 \mathrm{H}, t-\mathrm{Bu}), 6.82$ (s, 1H, 5-H), $8.43\left(\mathrm{~s}_{\mathrm{br}}, 1 \mathrm{H}, \mathrm{NH}\right) \mathrm{ppm}$. OH-signal could not be detected. ${ }^{13} \mathrm{C}$ NMR $\left(\mathrm{CDCl}_{3}, 126 \mathrm{MHz}\right): \delta=26.7,34.9$ (q, s, $t$-Bu), $120.1\left(\mathrm{q},{ }^{1} J_{\mathrm{CF}}=273 \mathrm{~Hz}, \mathrm{CF}_{3}\right), 108.9(\mathrm{~d}, \mathrm{C}-5), 138.4(\mathrm{q}$, $\left.{ }^{2} J_{\mathrm{CF}}=36.4 \mathrm{~Hz}, \mathrm{C}-6\right), 136.7,146.5,170.7$ (3 s, C-2, C-3, C-4) ppm. IR (KBr): 3490-3330 (O-H, N-H), 3100-3060 (=C-H), 2960-2870 (C-H), 1710-1580 (C=O, C=C $) \mathrm{cm}^{-1}$. $\mathrm{C}_{10} \mathrm{H}_{12} \mathrm{~F}_{3} \mathrm{NO}_{2}$ (235.2): calcd. C, 51.07; H, 5.14; N, 5.96; found: C, 50.87; H, 5.04; N, 5.81 .

\section{Conversion into pyridinediyl bis(perfluoro- alkanesulfonates), typical procedure}

Pyridine-3,4-diol 2c (100 mg, $0.425 \mathrm{mmol})$ was dissolved in dichloromethane $(4 \mathrm{~mL})$ and $\mathrm{Et}_{3} \mathrm{~N}(0.24 \mathrm{~mL}, 1.70 \mathrm{mmol})$ was 
added. The solution was cooled to $0{ }^{\circ} \mathrm{C}$ and $\mathrm{Tf}_{2} \mathrm{O}(0.29 \mathrm{~mL}$, $1.70 \mathrm{mmol}$ ) was added dropwise. After stirring for $1 \mathrm{~d}$ at room temperature the reaction mixture was diluted with water $(5 \mathrm{~mL})$ and extracted three times with dichloromethane $(5 \mathrm{~mL})$. The combined organic phases were dried over $\mathrm{Na}_{2} \mathrm{SO}_{4}$ and concentrated to dryness. Column chromatography on silica gel (hexane) afforded $190 \mathrm{mg}$ (90\%) of $\mathbf{3 b}$ as a colorless oil (volatile under high vacuum).

2-tert-Butyl-6-(trifluoromethyl)pyridine-3,4-diyl bistriflate (3b): ${ }^{1} \mathrm{H} \mathrm{NMR}\left(\mathrm{CDCl}_{3}, 500 \mathrm{MHz}\right): \delta=1.51(\mathrm{~s}, 9 \mathrm{H}, t-\mathrm{Bu}), 7.71$ (s, $1 \mathrm{H}, 5-\mathrm{H}) \mathrm{ppm} .{ }^{13} \mathrm{C} \mathrm{NMR}\left(\mathrm{CDCl}_{3}, 126 \mathrm{MHz}\right): \delta=29.5,40.0$ $(\mathrm{q}, \mathrm{s}, t-\mathrm{Bu}), 112.1\left(\mathrm{dq},{ }^{3} J_{\mathrm{CF}}=3.2 \mathrm{~Hz}, \mathrm{C}-5\right), 118.5,119.8(2 \mathrm{q}$, ${ }^{1} J_{\mathrm{CF}}=321 \mathrm{~Hz}$ each, OTf), $120.0\left(\mathrm{q},{ }^{1} J_{\mathrm{CF}}=275 \mathrm{~Hz}, \mathrm{CF}_{3}\right), 147.2$ $\left(\mathrm{q},{ }^{2} J_{\mathrm{CF}}=36.9 \mathrm{~Hz}, \mathrm{C}-6\right), 136.0,149.4,166.5$ (3 s, C-2, C-3, C-4) ppm. ${ }^{\mathrm{z} 19} \mathrm{~F} \mathrm{NMR}\left(\mathrm{CDCl}_{3}, 470 \mathrm{MHz}\right): \delta=-68.3\left(\mathrm{~s}, \mathrm{CF}_{3}\right)$, $-71.1,-72.4$ (2 s, OTf) ppm. IR (film): $v=3110-3080(=\mathrm{C}-\mathrm{H})$, 2980-2880 (C-H), 1600-1575 (C=C) cm ${ }^{-1} \cdot \mathrm{C}_{12} \mathrm{H}_{10} \mathrm{~F}_{9} \mathrm{NO}_{6} \mathrm{~S}_{2}$ (499.3): calcd. C, 28.86; H, 2.02; N, 2.81; found: C, 28.89; H, $1.68 ;$ N 2.87 .

\section{Sonogashira coupling reaction, typical procedure}

A mixture of pyridinediyl bistriflate $\mathbf{3 b}(245 \mathrm{mg}, 0.491 \mathrm{mmol})$, $\mathrm{Pd}\left(\mathrm{PPh}_{3}\right)_{4}$ (79 mg, $\left.0.069 \mathrm{mmol}\right), \mathrm{CuI}(9.4 \mathrm{mg}, 0.049 \mathrm{mmol})$, (triisopropylsilyl)acetylene (215 mg, $1.18 \mathrm{mmol})$ in DMF $(2.3$ $\mathrm{mL})$ and diisopropylamine $(1.2 \mathrm{~mL})$ was heated to $60^{\circ} \mathrm{C}$ for $4 \mathrm{~h}$ under an argon atmosphere. The mixture was allowed to cool to room temperature, diluted with brine $(5 \mathrm{~mL})$ and extracted three times with diethyl ether $(5 \mathrm{~mL})$. The combined organic phases were dried over $\mathrm{Na}_{2} \mathrm{SO}_{4}$ and concentrated to dryness. The residue was purified by column chromatography on silica gel (hexane) followed by HPLC to give $98 \mathrm{mg}$ (35\%) of $\mathbf{4 a}$ as a colorless oil (volatile under high vacuum).

2-tert-Butyl-6-(trifluoromethyl)-3,4-bis[(triisopropylsilyl)ethynyl] pyridine (4a): ${ }^{1} \mathrm{H}$ NMR $\left(\mathrm{CDCl}_{3}, 500 \mathrm{MHz}\right): \delta=1.09-1.17$ (m, 42H, TIPS), 1.56 (s, 9H, $t$-Bu), 7.51 (s, $1 \mathrm{H}, 5-\mathrm{H}) \mathrm{ppm} .{ }^{13} \mathrm{C}$ NMR $\left(\mathrm{CDCl}_{3}, 126 \mathrm{MHz}\right): \delta=11.4,11.6,18.71,18.74$ (2 d, 2 q, TIPS), 28.7, $40.0(\mathrm{q}, \mathrm{s}, t$-Bu), 81.6, 90.2, 102.6, $103.7(4 \mathrm{~s}$, $\mathrm{C} \equiv \mathrm{C}), 108.2(\mathrm{~s}, \mathrm{C}-4), 121.3\left(\mathrm{q},{ }^{1} J_{\mathrm{CF}}=274 \mathrm{~Hz}, \mathrm{CF}_{3}\right), 121.4(\mathrm{dq}$, $\left.{ }^{3} J_{\mathrm{CF}}=3.1 \mathrm{~Hz}, \mathrm{C}-5\right), 143.7\left(\mathrm{q},{ }^{2} J_{\mathrm{CF}}=34.9 \mathrm{~Hz}, \mathrm{C}-6\right), 137.4(\mathrm{~s}$, $\mathrm{C}-3), 170.9$ (s, C-2) ppm. ${ }^{19} \mathrm{~F} \mathrm{NMR}\left(\mathrm{CDCl}_{3}, 470 \mathrm{MHz}\right): \delta=$ $-68.4\left(\mathrm{~s}, \mathrm{CF}_{3}\right) \mathrm{ppm}$. IR (film): $v=2950-2860(=\mathrm{C}-\mathrm{H}, \mathrm{C}-\mathrm{H})$, 2145-2065 $(\mathrm{C} \equiv \mathrm{C}), 1750-1575(\mathrm{C}=\mathrm{C}) \mathrm{cm}^{-1}$. HRMS (ESI-TOF) calcd. for $\mathrm{C}_{32} \mathrm{H}_{53} \mathrm{~F}_{3} \mathrm{NSi}_{2}[\mathrm{M}+\mathrm{H}]^{+}$: 564.3663; found 564.3690 .

\section{Conversion to bisalkyne 5}

Pyridine derivative 4a (50 mg, $0.089 \mathrm{mmol})$ was dissolved in THF ( $2 \mathrm{~mL})$ and TBAF $(0.36 \mathrm{~mL}, 1 \mathrm{M}$ in THF, $0.356 \mathrm{mmol})$ was added at room temperature. After stirring for $1 \mathrm{~h}$ the reac- tion mixture was diluted with water $(3 \mathrm{~mL})$ and extracted three times with ethyl acetate $(3 \mathrm{~mL})$. The combined organic phases were dried over $\mathrm{Na}_{2} \mathrm{SO}_{4}$ and concentrated to dryness. Column chromatography on silica gel (hexane/ethyl acetate $=40: 1$ ) afforded $13 \mathrm{mg}(58 \%)$ of $\mathbf{5}$ as a colorless solid, mp $79-81{ }^{\circ} \mathrm{C}$.

2-tert-Butyl-3,4-diethynyl-6-(trifluoromethyl)pyridine (5): ${ }^{1} \mathrm{H}$ NMR $\left(\mathrm{CDCl}_{3}, 500 \mathrm{MHz}\right): \delta=1.54(\mathrm{~s}, 9 \mathrm{H}, t-\mathrm{Bu}), 3.57,3.92(2$ $\mathrm{s}, 2 \mathrm{H}, \mathrm{C} \equiv \mathrm{CH}), 7.56(\mathrm{~s}, 1 \mathrm{H}, 5-\mathrm{H}) \mathrm{ppm} .{ }^{13} \mathrm{C} \mathrm{NMR}\left(\mathrm{CDCl}_{3}, 126\right.$ $\mathrm{MHz}): \delta=28.7,39.9(\mathrm{q}, \mathrm{s}, t-\mathrm{Bu}), 79.5,79.7,86.5,92.5(2 \mathrm{~s}, 2 \mathrm{~d}$, $\mathrm{C} \equiv \mathrm{CH}), 120.1\left(\mathrm{dq},{ }^{3} J_{\mathrm{CF}}=2.8 \mathrm{~Hz}, \mathrm{C}-5\right), 121.1\left(\mathrm{q},{ }^{1} J_{\mathrm{CF}}=274\right.$ $\mathrm{Hz}, \mathrm{CF}_{3}$ ), 121.3 (q, $\left.{ }^{4} J_{\mathrm{CF}}=1.2 \mathrm{~Hz}, \mathrm{C}-4\right), 137.0$ (s, C-3), 144.5 (q, $\left.{ }^{2} J_{\mathrm{CF}}=35.3 \mathrm{~Hz}, \mathrm{C}-6\right), 171.0(\mathrm{~s}, \mathrm{C}-2) \mathrm{ppm} .{ }^{19} \mathrm{~F} \mathrm{NMR}\left(\mathrm{CDCl}_{3}\right.$, $470 \mathrm{MHz}): \delta=-68.4\left(\mathrm{~s}, \mathrm{CF}_{3}\right) \mathrm{ppm}$. IR $(\mathrm{KBr}): v=3305(\equiv \mathrm{C}-\mathrm{H})$, 3000-2850 (=C-H, C-H), 2225-2105 (C $\equiv \mathrm{C}), 1765-1575(\mathrm{C}=\mathrm{C})$ $\mathrm{cm}^{-1}$. HRMS (ESI-TOF) calcd. for $\mathrm{C}_{14} \mathrm{H}_{13} \mathrm{~F}_{3} \mathrm{~N}[\mathrm{M}+\mathrm{H}]^{+}$: 252.0995; found 252.1009.

\section{Supporting Information}

Supporting Information File 1 contains the supplementary data for compounds $\mathbf{2 d}, \mathbf{3 a}, \mathbf{3 c}-\mathbf{d}$ and $\mathbf{4 b}-\mathbf{c}$.

\section{Supporting Information File 1}

Supplementary data for compounds $\mathbf{2 d}, \mathbf{3 a}, \mathbf{3 c}-\mathbf{d}$ and $\mathbf{4 b}-\mathbf{c}$. [http://www.beilstein-journals.org/bjoc/content/ supplementary/1860-5397-6-42-S1.pdf]

\section{Acknowledgements}

Generous support of this work by the Deutsche Forschungsgemeinschaft, the Fonds der Chemischen Industrie and the BayerSchering Pharma AG is most gratefully acknowledged. We thank Dr. R. Zimmer for his help during preparation of this manuscript.

\section{References}

1. Müller, T. J. J.; Bunz, U. H. F., Eds. Functional Organic Materials; Wiley-VCH: Weinheim, Germany, 2007.

2. McKillop, A.; Boulton, A. J. In Comprehensive Heterocyclic Chemistry; Rees, C. W.; Katritzky, A. R., Eds.; Pergamon: Oxford, U.K., 1984; Vol. 2, pp 67-134.

3. Jones, G. In Comprehensive Heterocyclic Chemistry; McKillop, A., Ed.; Pergamon: Oxford, U.K., 1996; Vol. 5, pp 167-243.

4. Spitzner, D. Product Class 1: Pyridines. In Six-Membered Hetarenes with One Nitrogen or Phosphorus Atom; Black, D. StC., Ed.; Science of Synthesis, Vol. 15; Thieme: Stuttgart, Germany, 2004; pp 11-284.

5. Christinat, N.; Scopelliti, R.; Severin, K. J. Org. Chem. 2007, 72, 2192-2200. doi:10.1021/jo062607p

6. Dehkordi, L. S.; Liu, Z. D.; Hider, R. C. Eur. J. Med. Chem. 2008, 43, 1035-1047. doi:10.1016/j.ejmech.2007.07.011

7. Negwer, M. Organic-Chemical Drugs and Their Synonyms, 7th ed.; Akademie-Verlag: Berlin, Germany, 1994. 
8. Reissig, H.-U.; Schade, W.; Okala Amombo, M. G.; Pulz, R.; Hausherr, A. Pure Appl. Chem. 2002, 74, 175-180. doi:10.1351/pac200274010175

9. Kaden, S.; Reissig, H.-U. Org. Lett. 2006, 8, 4763-4766. doi:10.1021/ol061538y

10. Sörgel, S.; Azap, C.; Reissig, H.-U. Org. Lett. 2006, 8, 4875-4878. doi:10.1021/ol061932w

11. Brasholz, M.; Reissig, H.-U.; Zimmer, R. Acc. Chem. Res. 2009, 42, 45-56. doi:10.1021/ar800011h

12. Pfrengle, F.; Lentz, D.; Reissig, H.-U. Angew. Chem. 2009, 121, 3211-3215. doi:10.1002/ange.200805724 Angew. Chem., Int. Ed. 2009, 48, 3165-3169. doi:10.1002/anie.200805724

13. Lechel, T.; Lentz, D.; Reissig, H.-U. Chem.-Eur. J. 2009, 15 , 5432-5435. doi:10.1002/chem.200900386

14. Lechel, T.; Möhl, S.; Reissig, H.-U. Synlett 2009, 1059-1062. doi:10.1055/s-0028-1088220

15. Pfrengle, F.; Reissig, H.-U. Chem. Soc. Rev. 2010, 39, 549-557. doi:10.1039/b914356d

16. Lechel, T.; Reissig, H.-U. Eur. J. Org. Chem. 2010, 2555-2564. doi:10.1002/ejoc.201000056

17. Lechel, T.; Reissig, H.-U. Pure Appl. Chem., in press.

18. Flögel, O.; Dash, J.; Brüdgam, I.; Hartl, H.; Reissig, H.-U. Chem.-Eur. J. 2004, 10, 4283-4290. doi:10.1002/chem.200400322

19. Dash, J.; Lechel, T.; Reissig, H.-U. Org. Lett. 2007, 9, 5541-5544. doi:10.1021/ol702468s

20. Lechel, T.; Dash, J.; Brüdgam, I.; Reissig, H.-U. Eur. J. Org. Chem. 2008, 3647-3655. doi:10.1002/ejoc.200800398

21. Eidamshaus, C.; Reissig, H.-U. Adv. Synth. Catal. 2009, 351 , 1162-1166. doi:10.1002/adsc.200800789

22. Lechel, T.; Dash, J.; Hommes, P.; Lentz, D.; Reissig, H.-U. J. Org. Chem. 2010, 75, 726-732. doi:10.1021/jo9022183

23. Lechel, T.; Dash, J.; Eidamshaus, C.; Brüdgam, I.; Lentz, D.; Reissig, H.-U. Org. Biomol. Chem., in press.

24. CCDC-766558 (for 2c) contains the supplementary crystallographic data. These data can be obtained free of charge from The Cambridge Crystallographic Data Centre via www.ccdc.cam.ac.uk/data_request/cif.

25. Högermeier, J.; Reissig, H.-U. Adv. Synth. Catal. 2009, 351, 2747-2763. doi:10.1002/adsc.200900566

26. Sonogashira, K. In Handbook of Organopalladium Chemistry for Organic Synthesis; Negishi, E.-i.; de Meijere, A., Eds.; Wiley: New York, 2002; pp 493-529.

27. Marsden, J. A.; Haley, M. M. Cross-Coupling Reactions to sp Carbon Atoms. In Metal-Catalyzed Cross-Coupling Reactions, 2nd ed.; de Meijere, A.; Diederich, F., Eds.; Wiley-VCH: Weinheim, Germany, 2004; pp 317-394.

28. Negishi, E.-i.; Anastasia, L. Chem. Rev. 2003, 103, 1979-2017. doi:10.1021/cr020377

29. Chinchilla, R.; Nájera, C. Chem. Rev. 2007, 107, 874-922. doi:10.1021/cr050992x

30. Doucet, H.; Hierso, J.-C. Angew. Chem. 2007, 119, 850-888. doi:10.1002/ange.200602761

Angew. Chem., Int. Ed. 2007, 46, 834-871. doi:10.1002/anie.200602761

31. Forrest, S. R.; Thompson, M. E., Eds. Organic Electronics and Optoelectronics. Chem. Rev. 2007, 107, 923-1386. doi:10.1021/cr0501590

32. Müllen, K.; Scherf, U., Eds. Organic Light-Emitting Devices: Synthesis, Properties and Applications; Wiley-VCH: Weinheim, Germany, 2006.
33. Yamaguchi, Y.; Tanaka, T.; Kobayashi, S.; Wakamiya, T.; Matsubara, Y.; Yoshida, Z.-i. J. Am. Chem. Soc. 2005, 127, 9332-9333. doi:10.1021/ja051588i

34. Basak, A.; Mandal, S.; Bag, S. S. Chem. Rev. 2003, 103, 4077-4094. doi:10.1021/cr020069k

35. Choy, N.; Blanco, B.; Wen, J.; Krishan, A.; Russell, K. C. Org. Lett. 2000, 2, 3761-3764. doi:10.1021/ol006061j

\section{License and Terms}

This is an Open Access article under the terms of the Creative Commons Attribution License

(http://creativecommons.org/licenses/by/2.0), which permits unrestricted use, distribution, and reproduction in any medium, provided the original work is properly cited.

The license is subject to the Beilstein Journal of Organic Chemistry terms and conditions:

(http://www.beilstein-journals.org/bjoc)

The definitive version of this article is the electronic one which can be found at: doi:10.3762/bjoc. 6.42 